\title{
Three years continuous record of the Earth's magnetic field at Concordia Station (DomeC, Antarctica)
}

\author{
Aude Chambodut $\left({ }^{1}\right)$, Domenico Di Mauro $\left({ }^{2}\right)$, Jean-Jacques Schott $\left({ }^{1}\right)$, Pascal Bordais $\left({ }^{3}\right)$, \\ Lucia Agnoletto $\left({ }^{4}\right)$ and Pietro di Felice $\left({ }^{4}\right)$ \\ (') Ecole et Observatoire des Sciences de la Terre, Strasbourg, France \\ (') Istituto Nazionale di Geofisica e Vulcanologia, Roma, Italy \\ $\left(^{3}\right)$ Institut Polaire Français Paul Emile Victor, Plouzané, France \\ $\left.{ }^{4}\right)$ C.R. ENEA-Casaccia, S.M. di Galeria (RM,) Italy
}

\begin{abstract}
The magnetic observatory deployed at DomeC, Antarctica, in the French-Italian base known as Concordia has now been permanently running for more than three years. This paper focuses on these long-term results which are more relevant for an observatory intended to provide absolute values of the field. The problems which emerged in this fairly long record are discussed and solutions suggested to upgrade the observatory to the standards of an absolute one (i.e. Intermagnet standards).
\end{abstract}

Key words Antarctica-DomeC-magnetic observatory - continuos records

\section{Introduction}

The DomeC site was selected in 1974 to start research activities under the framework of the International Glaciology Project (IAGP). A shallow coring, joint French/ American program was conducted at the beginning of the 1980's with the support of the National Science Foundation. The camp was abandoned after the end of the coring (to a depth of about 900m), which was hampered by three aircraft crashes.

At the beginning of the 1990's a French Italian venture planned to build a permanent scientific base. The DomeC site was chosen again,

Mailing address: Dr. Aude Chambodut, Ecole et Observatoire des Sciences de la Terre 5, rue Descartes 67084, Strasbourg Cedex, France; e-mail: Aude.Chambodut@unistra.fr mainly because of the thickness of the ice cap and the low atmospheric water vapour content. The first remarkable activity was a long ice core drilling, which should help deciphering the past climate of our planet over a large time span. The coring lasted from 1997 to 2005 with the aim at drilling through the whole ice cap.

Several kinds of scientific activities have now started beside the glaciology project: atmosphere analysis, astronomy, geophysics (seismology and geomagnetism).

There are currently a dozen magnetic observatories running on the Antarctic continent (Schott and Rasson, 2007), if we term observatories those providing base line control. The quality of this control is variable, ranging from episodic measurements performed during summer campaigns to measurements made regularly throughout the year at the staffed bases hosting qualified observers. Thus, as shown in fig. 1, only four observatories - Dumont d'Urville (DRV), Scott Base (SBA), Mawson (MAW) and Argentine Island (AIA) - provide absolute values according to modern standards like those 


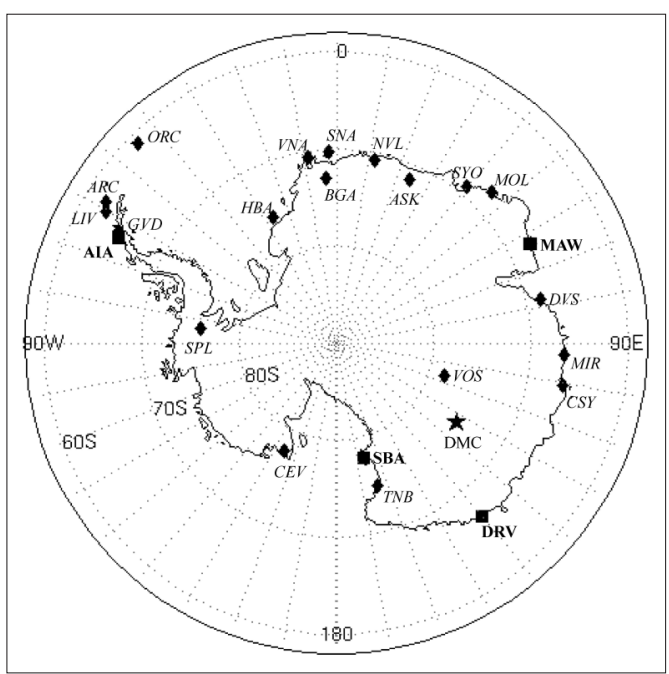

Fig. 1. Location of magnetic observatories in Antarctica. Square: Intermagnet observatories; diamond: observatories with various status; star: DomeC observatory.

stated by Intermagnet. From this point of view, the lack of balance with the Northern hemisphere at similar latitudes is striking. In addition, each of DRV, SBA and MAW observatories is located on extremely magnetized basements, causing severe observatory biases which are a drawback to be taken into account in global or regional models (e.g. Mandea and Langlais, 2002). They are also submitted to coast effects which influence the power spectrum at diurnal frequencies and beyond.

DomeC (hereafter quoted DMC) is operated jointly by Ecole et Observatoire des Sciences de la Terre (EOST), Strasbourg, France, and by Istituto Nazionale di Geofisica e Vulcanologia (INGV), Rome, Italy, with the logistic and partly financial support of the French Institution Institut Polaire Français Paul Emile Victor (IPEV) and the Italian Institution Ente per le Nuove Tecnologie, l'Energia e l'Ambiente (ENEA). The aim is to operate DMC in accordance to the accuracy requirements stated by Intermagnet and hence, to provide data constraining helpfully global and regional models of the main field and its secular variation (De
Santis et al., 2002; Torta et al., 2002; GayaPiqué et al., 2006). Regarding the external field, DMC is situated inside the polar cap, close to the invariant pole and the geomagnetic South pole. The auroral zones and polar caps are areas where the spatial variation of phenomena like magnetic storms, substorms, and pulsations is sharp. Hence, their study requires the availability of a dense network of stations. Once again, this is the case in the Northern hemisphere. In the Southern hemisphere, VOS, Casey (CSY), DMC, DRV, SBA and Terra Nova Bay (TNB) would build up a fairly dense network in the particular region mentioned above. The paper published by Lepidi et al., 2003, based upon data from TNB and DMC, highlights the interest for DMC from external point of view.

Some other specifications of the observatory may be found in Schott et al. (2005).

\section{Equipment and protocol of measure- ments}

The description of the observatory equipment was already given in Schott et al. (2005). It is provided again hereafter with some updates, for the sake of completeness.

The observatory is equipped with standard instruments for continuous three-component field variation recording and absolute measurements. The field variations are recorded with a suspended triaxial fluxgate magnetometer (FGE type) especially manufactured by the Danish Meteorological Institute for low temperatures, with suitable damping silicon oil and silicon connection cables. It is put on a pillar penetrating about one meter into the ice. Although the horizontal component is weak (around 10400nT), the variometer is oriented with respect to the local magnetic meridian. The technical specifications are: dynamic range $\pm 4000 \mathrm{nT}$; resolution $0.2 \mathrm{nT}$; temperature coefficient of sensor lower than $0.2 \mathrm{nT} /{ }^{\circ} \mathrm{C}$; temperature coefficient of electronics lower than $0.1 \mathrm{nT} /{ }^{\circ} \mathrm{C}$; band pass $\mathrm{DC}$ to $1 \mathrm{~Hz}$; sampling rate 1Hz. An Overhauser proton magnetometer SM90R records the field intensity at a sampling rate of $0.2 \mathrm{~Hz}$. Both instruments are installed in a cave, two meters deep, situated beneath the 
shelter containing the electronics and acquisition system. The acquisition system was built up by INGV. It comprises mainly a 24 bit $A D$ converter and a PC driving both the AD converter and the SM90R. Accurate timing is provided by GPS signal. A second PC connected to the acquisition displays a control board and current records. Electronics and acquisition are located inside a thermally controlled box. Data are transmitted to the main building via a $\mathrm{Wi}-\mathrm{Fi}$ connection. One minute records and absolute measurements are sent manually by the observer, weekly both to EOST and INGV. Thus, a fairly timely remote control of the observatory is made possible.

The absolute measurements are performed on a pillar made with a polyethylene tube, standing temperatures down to less than $-40^{\circ} \mathrm{C}$. Its dimensions are: length $3 \mathrm{~m}$, diameter $40 \mathrm{~cm}$, thickness $2.27 \mathrm{~cm}$. It penetrates $1.75 \mathrm{~m}$ into the ice. It is located inside a shelter which is continuously heated at about $10^{\circ} \mathrm{C}$. The absolute measurements consist of $\mathrm{D}$, I measurements with a D/ I flux non-magnetic theodolite and F measurements performed manually with a second Overhauser proton magnetometer SM90R. The theodolite is a Zeiss 010A type with reading in grades, 0.5 second of arc resolution. The sensor and driving electronics are Bartington devices with $0.1 \mathrm{nT}$ resolution. An azimuth mark was fixed onto the variometer shelter, about $25 \mathrm{~m}$ distant. Its true North bearing was determined from sun observations (Newitt et $a l .$, 1996). Due to magnetic activity, declination and inclination are measured using a close-tozero method (Jankowski and Sucksdorff, 1996) and all readings are reduced to a common time, nearly the mean time of the whole sequence time span.

To summarize, three component field variations are recorded at $1 \mathrm{~Hz}$ sampling rate. Intensity is recorded at $0.2 \mathrm{~Hz}$ sampling rate. Both kinds of data are filtered according to Intermagnet recommendations and resampled at one minute sampling rate.

The results obtained during three preliminary summer campaigns achieved in December 1999-January 2000, December 2001 and December 2003-January 2004 were published in Schott et al. (2005). Therefore, this paper fo- cuses on the more or less continuous records beginning in February 2005 when the base (named Concordia) opened permanently. Although, the observatory is at the moment operated by non dedicated and non qualified observers, we believe that the data quality is good enough to be of interest for the scientific community.

\section{Base lines}

\subsection{Adopted base lines}

Let us recall that one aim with base line measurements is to compute absolute values of the field components. Thus, a smooth continuous series $\overrightarrow{\boldsymbol{B}}_{b l}(t)$, where the subscript $b l$ stands for base line, has to be estimated from the set of spot values $\overrightarrow{\boldsymbol{B}}_{b l}\left(t_{i}\right)_{i=1, K K N}$ by some fitting method. $\overrightarrow{\boldsymbol{B}}_{b l}\left(t_{i}\right)$ may be represented either by its Cartesian components in the geographic reference frame, or, regarding the horizontal components, by its traditional polar form $\mathrm{H}\left(\mathrm{t}_{\mathrm{i}}\right), \mathrm{D}\left(\mathrm{t}_{\mathrm{i}}\right)$. In our case, according to Section 2, this latter form is the immediate outcome of the measurement protocol. Current choices for the fitting are either parametric (for instance a set of polynomials orthogonal over the set of sampled times) or non parametric (for example cubic smoothing splines).

Unfortunately, during the 2006-2007 summer campaign, a very inappropriate decision was taken to deploy an electric power line close to the shelters of the observatory, despite the agreement upon a clean area around the observatory. As a result, no absolute measurements could be performed during most of the year 2007 due to the disturbance from this power line. Luckily, it was removed during the 20072008 summer campaign and proper measurements could be resumed since the end of December 2007. The very last measurements were incorporated into the data set in order to better constrain the fitting (as can be noted in fig. 2, the range for adopted base line computation may be strictly included in the range of available data used for the computation of spline functions or orthogonal polynomials. In general, this avoids boundary effects. In addition, in the present study, base lines are needed only till 


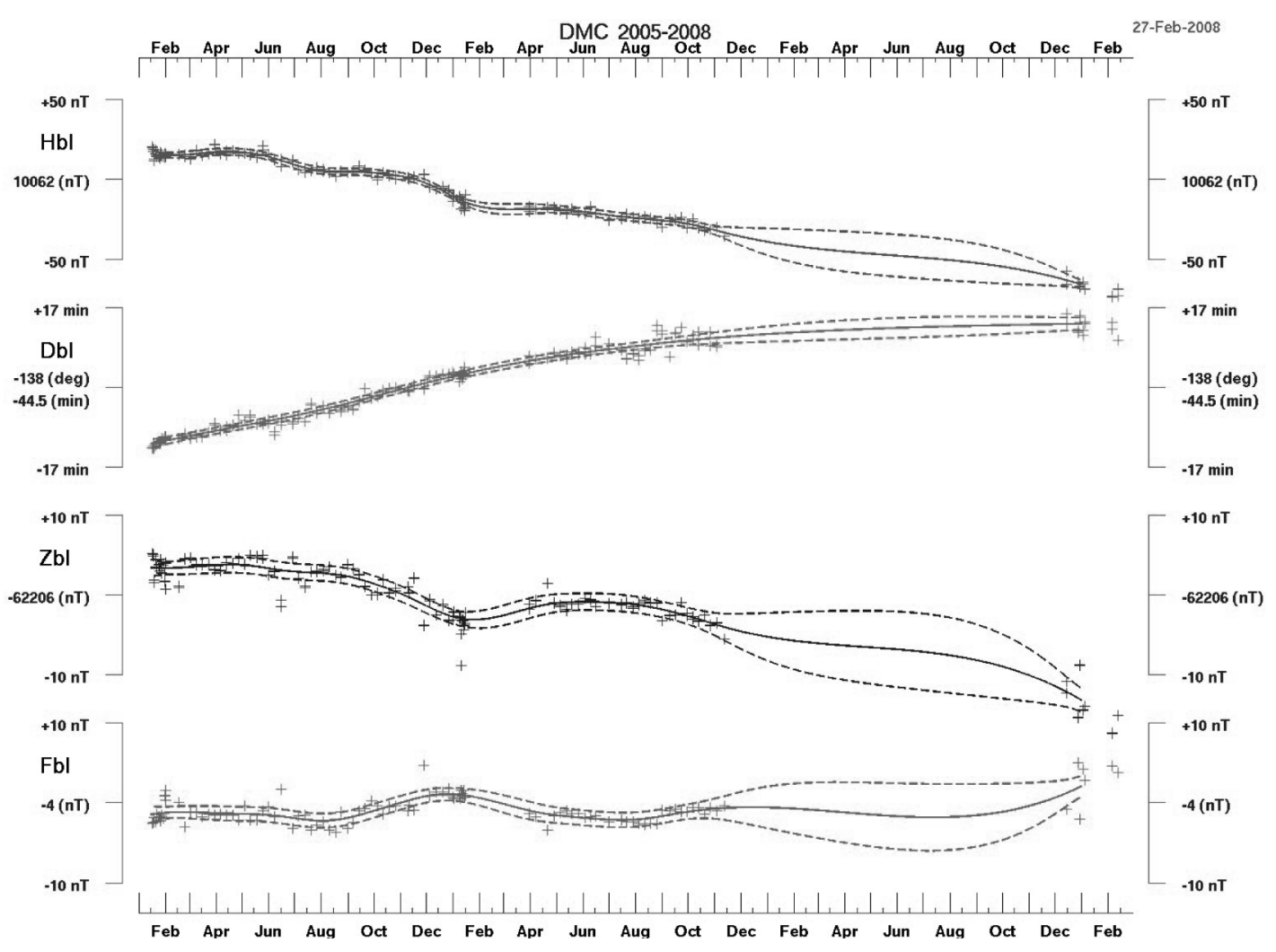

Fig. 2. 2005-2007 base line fitting using cubic splines for the horizontal $\mathrm{H}$ component, declination D, vertical component $\mathrm{Z}$, total field difference $\mathrm{F}$ between the proton magnetometer measurements on the absolute pillar and variometer measurements on the absolute pillar and variometer cave respectively. Crosses: spot values; solid lines: spline adjustment; dashed lines: limits of the $95 \%$ confidence interval.

the end of December 2007). Due to the huge gap in the series, the fitting is not straightforward. This is an important issue because the final values depend of course on this fitting and hence the time variation of the field. We have tested both a spline and a low degree (up to 3 ) orthogonal polynomials fitting. Figures 2 and 3 show the results for the spline fitting. Figure 2 shows the spot values as well as the fitting for $\mathrm{H}, \mathrm{D}, \mathrm{Z}$ and $\mathrm{F}$ which is the total field difference between the absolute pillar and the variometer cave. As expected, the small values of $F_{b l}$ reflect the absence of crustal contamination. The non zero mean value is due to the various equipments stored in the variometer house. Figure 3 displays some statistical aspects of the goodness of fit, with statistical parameters derived using the method described by Silverman (1985). One well-known criterion (e.g. Seber, 1977) is provided by the time series of the residuals. Figure 3 shows that they are fairly randomly distributed, with a distribution consistent with the underlying Gaussian assumption. However, the oscillations displayed in fig. 2 for the year 2007 are evidently questionable although they can be seen also on better sampled parts of the curves. They may account, at least regarding the fluxgate vector magnetometer, for an annual variation of the magnetometer (sensors and electronics) and /or acquisition temperature, although the temperature variation is not well documented (see below, Section 4). 

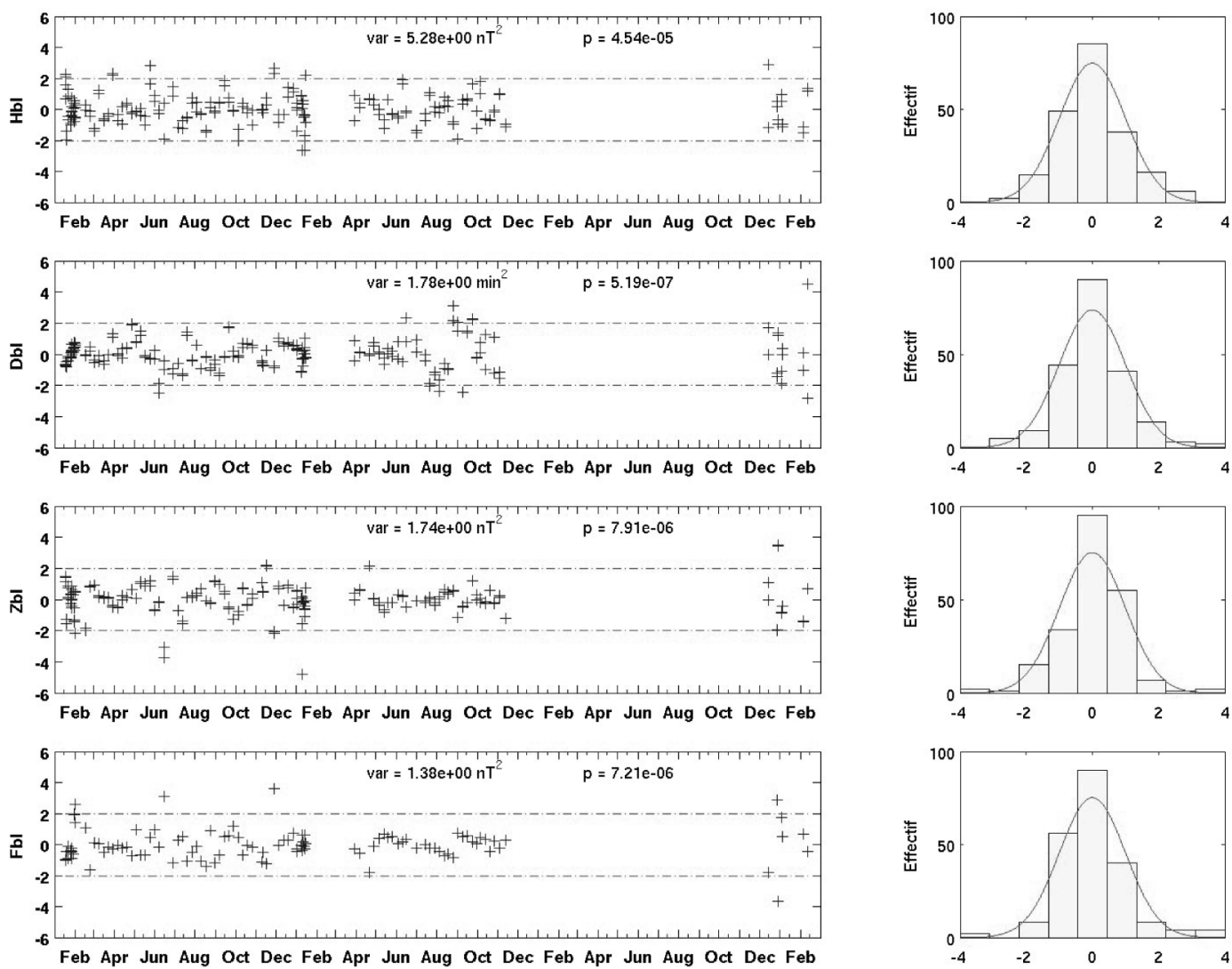

Fig. 3. Left part: studentized (dimensionless) residuals versus time for each component of the base lines shown on fig. 2 var stands for the variance of the statistical model, $\mathrm{p}$ for the smoothing factor. Right part: distribution of studentized residuals compared to a Normal Gaussian distribution.

As to the total field difference, $\mathrm{F}_{\mathrm{lb}}$, derived from proton magnetometers, thermal effects cannot not be precluded either (Sapunov et al., 2001) all the more as so, the sensor is working at unusually low temperatures. In addition, regarding the whole set of base lines, we also have to keep in mind the annual disturbances brought about during the summer campaigns (for instance, these disturbances may account for the scattering observed on the very last measurements). A more conservative fitting is provided by the low degree orthogonal polynomials as can be seen in fig. 4. Despite the time distribution of residuals displayed in fig. 5 contains underfitting features, we will adopt this base line adjustment for the computation of the absolute values. The discussion given in the next Section is based upon this model as well.

\subsection{Base line drift}

Figures 2 and 3 show a conspicuous drift of the base lines over the whole time span. We have tentatively explained this drift by a progressive rotation of the magnetometer, assuming that the sensors are mutually orthogonal and the base lines constant in the sensor reference frame (in this frame, they are merely the bias and offset fields). Writing $\underline{B}_{b l}^{G}(t)$ the base line vector at time $t$ in the geographic reference frame, we have estimated the rotation matrix 


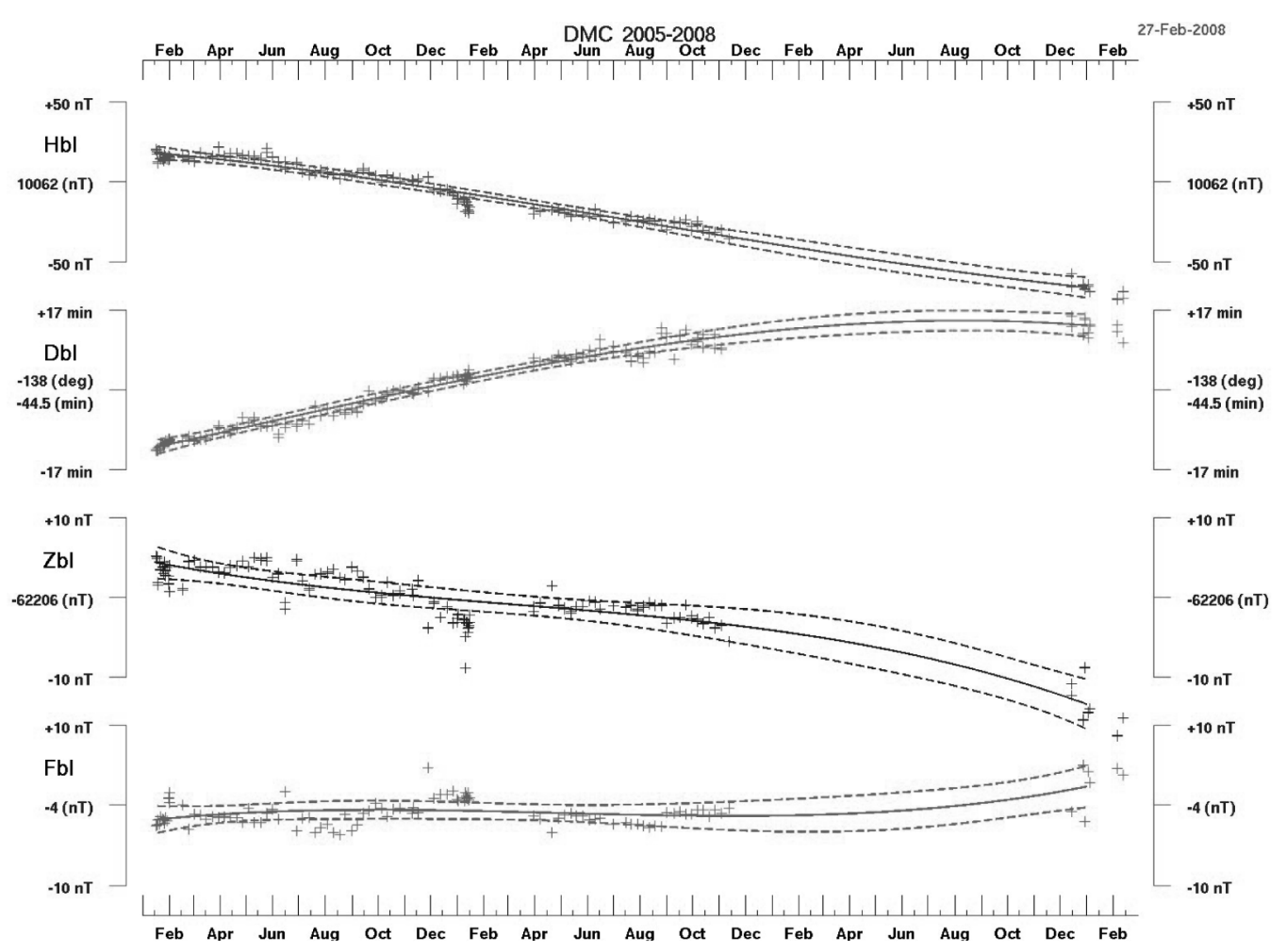

Fig. 4. Same as fig. 2, apart from the fitting model wich is a sequence of orthogonal polynomials up to degree 3. See text for further comments.

$\underline{R}_{=}(t)$ which maps $\underline{B}_{b l}^{G}\left(t_{0}\right)$ onto $\underline{B}_{b l}^{G}(t) . \underline{B}_{b l}^{G}(t)$ and $\underline{B}_{b l}^{G}\left(t_{0}\right)$ are the adjusted base lines at times $\mathrm{t}$ and $\mathrm{t}_{0}$, described in the previous Section. $\mathrm{t}_{0}$ is some time origin, taken as the time of the first value of the series $\underline{B}_{b l}^{G}(t)$ in the range 20052007. The next approximation we made was to assume that the orientation of the sensors was known at time $t_{0}$. This is of course not true, although a common practice, with magnetometers aligned in the local geomagnetic reference frame, is to assimilate the direction of the $\mathrm{H}$ sensor to the base line declination, and to assume that the Z-sensor is actually vertical. However, this assumption will only influence the actual drift of the sensors, not the estimation of the rotation matrix, which anyway requires a further constraint. Indeed, the problem of computing the matrix $\underline{R}_{1}(t)$ knowing only a pair of vectors $\underline{B}_{b l}^{G}\left(t_{0}\right)$ and $\underline{B}_{b l}^{G}(t)$ has an infinite number of solutions. Among this set, we have selected the rotation having the smallest angle.

The results are shown in figs. 6 and 7. Figure 6 shows the paths followed by each of the three sensor orientations, with a time step of one month. In every case, somewhat unexpectedly, the movement is mainly in vertical planes, with an increasing dip for sensors $\mathrm{H}$ (negative) and $\mathrm{D}$ (positive), whereas the Z-sensor moves away from the vertical line. Figure 7 shows that the rotation vector is essentially horizontal, but that its axis itself rotates counter-clockwise. The rotation angle varies linearly with time for roughly the years 2005 and 2006, but afterwards exhibits a bending which indicates that the drift seems to decrease. The causes of the drift are either instrumental of environmental. Let us recall that the magnetometer is suspend- 

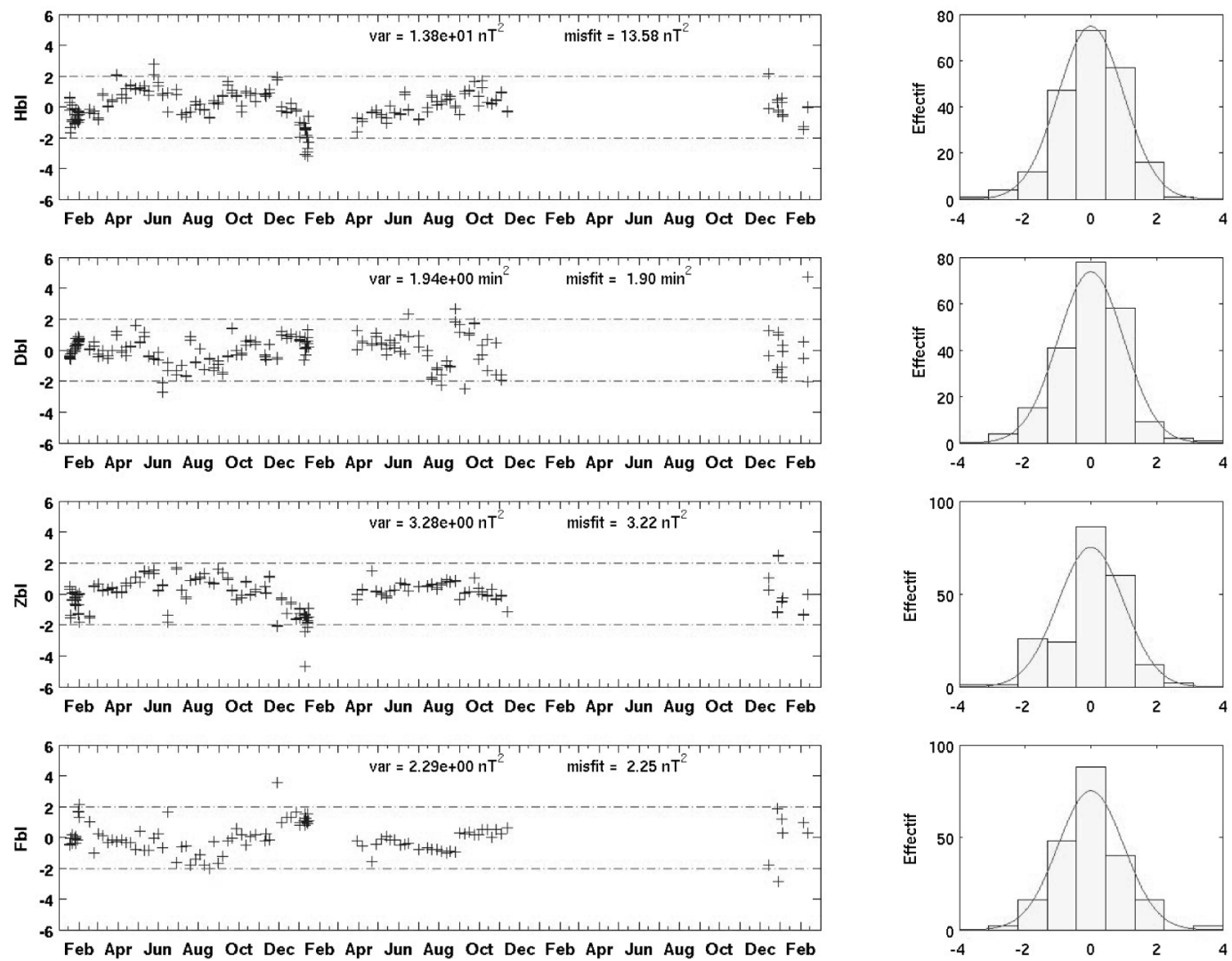

Fig. 5. Same as fig. 3 apart from the misfit parameter which is the usual square misfit in linear regression models.

ed. According to the technical specifications, the suspension device should compensate tilts up to $0.5^{\circ}$, that is in a range significantly larger than the estimated dip of the sensors. However, the proper working of the suspension assumes that the damping oil is not too viscous (or even frozen up). The choice of the damping oil assumed temperatures not lower than around $-40^{\circ}$ Celsius. It turns out that the winter temperatures are lower (see Section 4). Thus we cannot preclude a hardening of the oil. Another source of drift might be some unexpected strain of the brace plates which support the magnetometer. However, any drift due to either of these effects should have stopped long ago, because the magnetometer was installed in 1999. On the other hand, if we suspect some movement of the pillar, the magnetometer has to be rigidly fixed to its housing, so that this assumption, likewise, implies that the suspension is frozen in somehow. We should also keep in mind the assumption which the estimation of the rotation relies upon, namely the absence of drift due to the bias fields, and which is not supported by any direct evidence. However, on one hand, we have never noticed such a large drift with FGE magnetometers, though in less tough conditions, and, on the other hand, the very small heterogeneity between absolute and variometer shelters, as assessed from $F_{\mathrm{lb}}$ range, makes the drift of $\mathrm{D}$ base line inconsistent with a mere drift in $\mathrm{H}$ bias field. Anyway, further inquiries are necessary in order to suggest a plausible reason for the drift. 


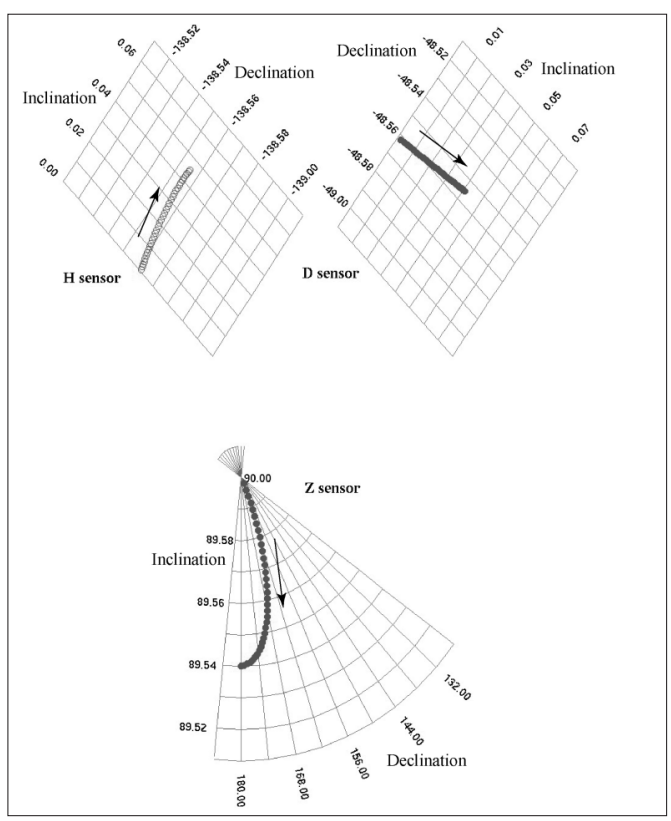

Fig. 6. Drift of the vector magnetometer sensors over the time span 2005-2007. H is nearly horizontal, close to the magnetic North direction; D is nearly horizontal, close to the magnetic East direction; $\mathrm{Z}$ is close to vertical. The time step is one month; the angles are expressed in degrees and minutes. Full circles: positive inclinations; open circles: negative inclinations.

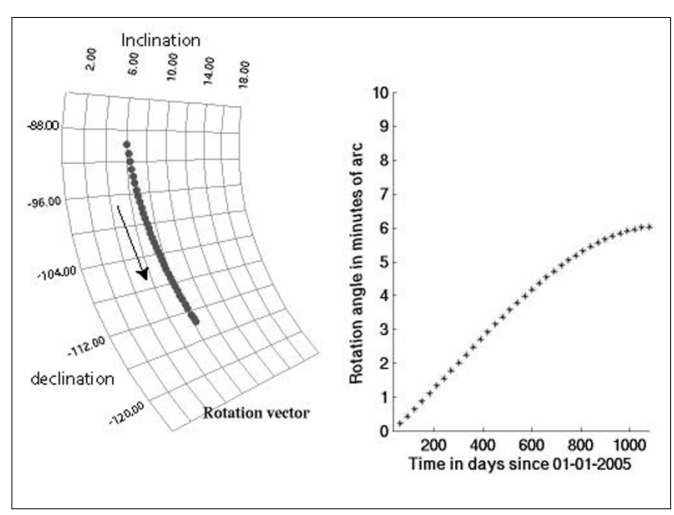

Fig. 7. Left: drift of the rotation vector accounting for the assumed rotation of the magnetometer sensors over the time span 2005-2007. Right: rotation angle versus time. See Section 3.2 for further explanations on the meaning of the rotation.

\section{Temperature records}

Temperatures have been recorded since January 2006 with small data loggers, independently from the acquisition device itself. One is put in the cave containing the magnetometer, about one meter above the bottom, the second one is located in the box housing the electronics and acquisition system. Unfortunately, only piecewise records are available, but nevertheless they are meaningful enough to illustrate the difficulty to fairly control the temperature variations.

The upper curve in fig. 8 shows hourly mean temperature variations in the vicinity of the electronics and acquisition device. The unexpected high values reached in the first part of the year 2006 are due to the box being closed by an insulating door. Even with the heating supply switched off, the heat dissipated by the various pieces of equipment was efficient enough to make the temperature rise to surprising levels. By mid-June, the door was left open and, as a result, the temperature dropped to around $10^{\circ} \mathrm{C}$. The two large drops noticeable around February 10 and September 25 are due to power supply interruptions. Most of the remaining jumps presumably reflect the temperature gradient inside the variometer house, as the temperature data logger was not exactly put on the same place after each data download. The small oscillations occurring mainly in the first and last part of the curve are daily variations not fully understood.

The lower curve in fig. 8 displays a short sequence of hourly mean temperature recorded in the cave. It shows that the temperature variation is unexpectedly large, all the more as the data logger fails to record temperatures lower than $-44^{\circ} \mathrm{C}$. Thus, unfortunately, we have so far no idea of the full range of variations. We refer to the previous section for the consequences this large range of excursion and decrease to unexpected low temperatures might have upon the base lines.

\section{Magnetic field daily means}

The purpose of this paper is to focus on the long-term change of the magnetic field. Daily variations are a good compromise for showing it along with some features of the external field. 


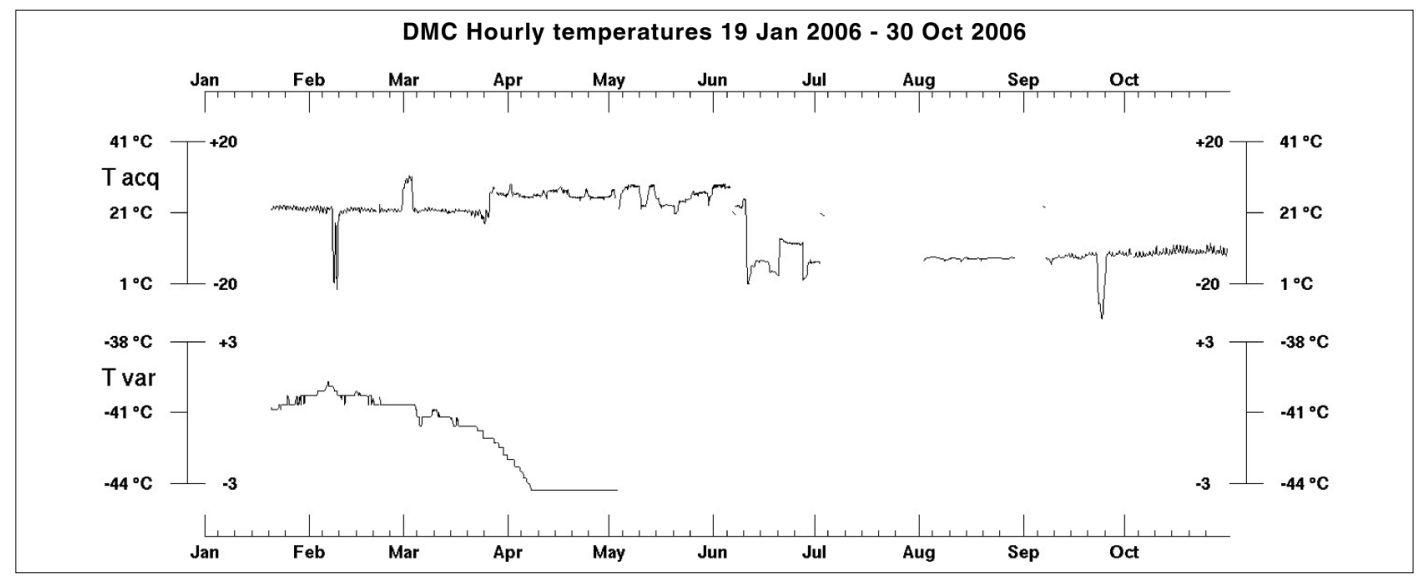

Fig. 8. Top: Hourly mean temperature record in the box housing the instrument electronics and acquisition device. There are mainly two parts, before and after the door of the box was left open (mid June 2006 onwards). See Section 4 for details regarding the additional jumps and peaks. Bottom: Hourly mean temperature record in the cave, close to the magnetometer. The temperature drops to values lower than $-44^{\circ} \mathrm{C}$, which is the lower limit of the data logger recording range.

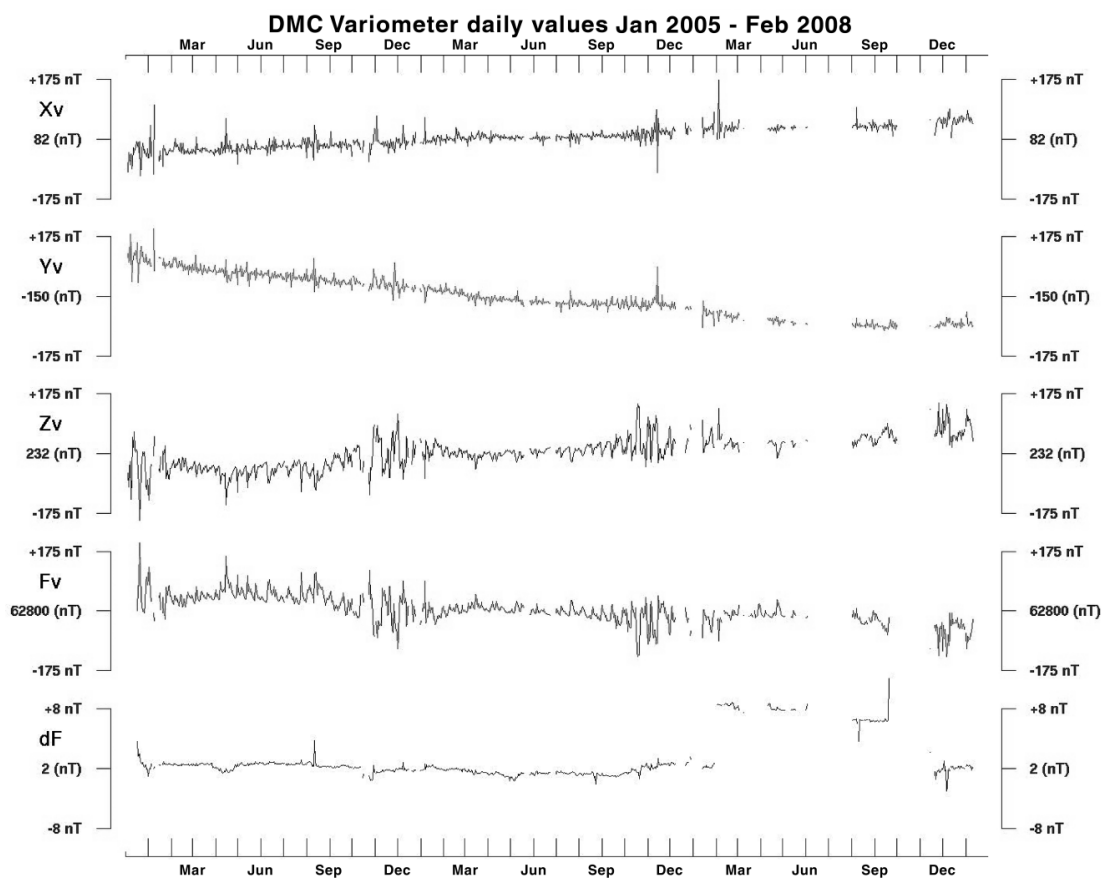

Fig. 9. Daily mean values of the magnetometer outputs. $\mathrm{Xv}, \mathrm{Yv}, \mathrm{Zv}$, stand for the components measured in the sensor reference frame (close to the local magnetic reference frame). Fv is the total field recorded by the proton magnetometer. $\mathrm{dF}$ is the difference $\mathrm{F}_{\text {recorded }}$ minus $\mathrm{F}_{\text {computed }}$ where $\mathrm{F}_{\text {computed }}$ is the intensity of the field resulting from the combination of the magnetometer outputs and the base lines. The jump of $\mathrm{dF}$ in the range March $1-$ mid-September 2007 is due to a move of the sensor of the scalar magnetometer. 


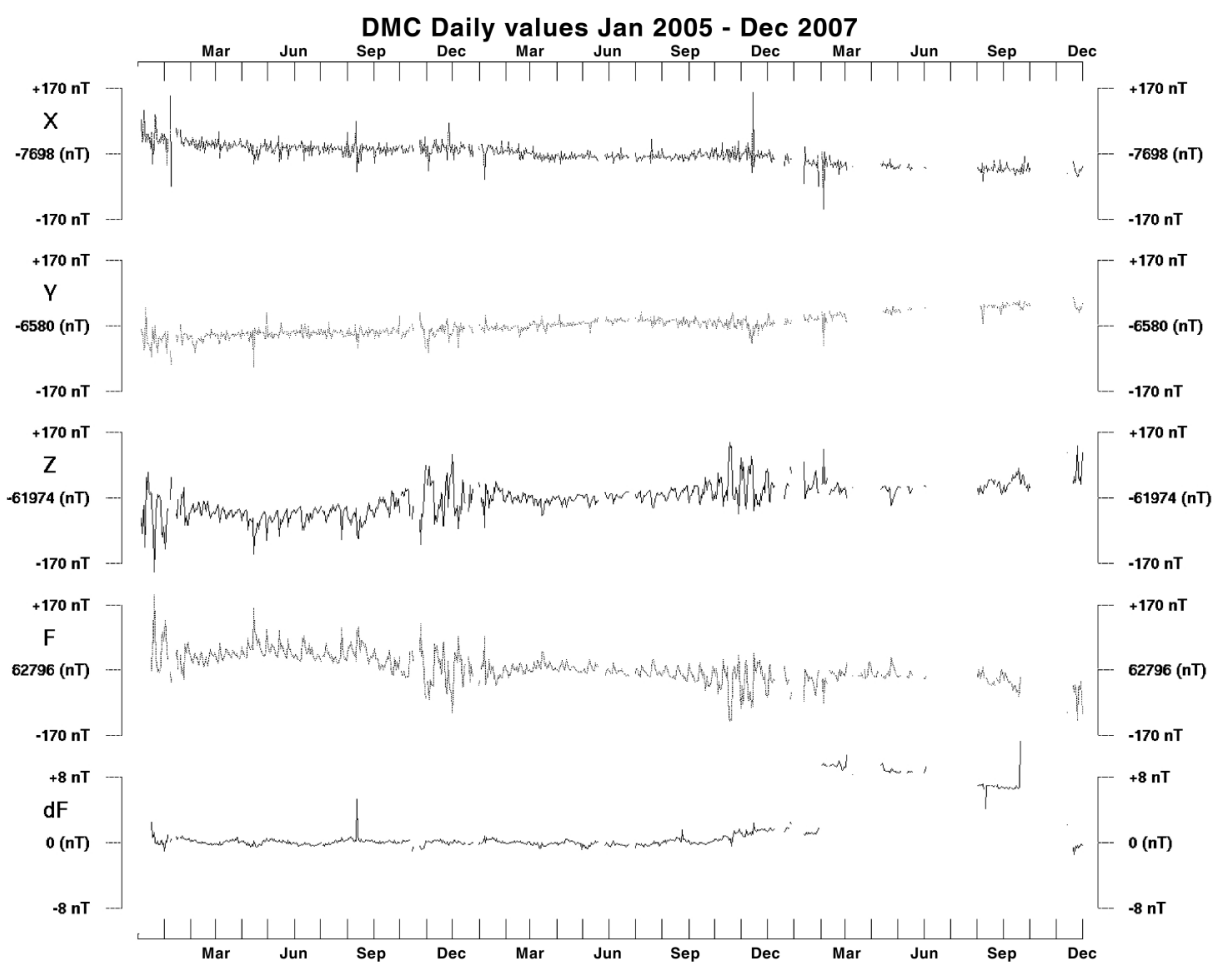

Fig. 10. Daily mean values of the absolute field. $\mathrm{X}, \mathrm{Y}, \mathrm{Z}$ are the components in the geographical reference frame. $\mathrm{F}$ and $\mathrm{dF}$ are equivalent to $\mathrm{Fv}$ and $\mathrm{dF}$, respectively, on fig. 9.

Regarding the daily variations, we refer to the work published by Cafarella et al. (2007) which describes some peculiarities of this variation in connection with IMF conditions. Figures 9 and 10 highlight clearly the seasonal variation due to large variation of the ionospheric conductivity controlled by the solar radiation.

As to the secular variation, the comparison between figs. 9 (field variation without base line correction) and 10 (with base lines added) illustrates the difference between an automatic observatory and an observatory providing absolute field components. The long-term correction afforded by the base line control is particularly striking in the current situation where a strong base line drift prevails over the natural secular variation. Besides, figures 9 and 10 display numerous data gaps in 2007 due to acquisition failures. The acquisition device is not protected against power supply breakdowns which occurred frequently during these three years of permanent working of the base. The incorporation of an uninterruptible power supply in April did not improve the working much. The repetition of power supply failures probably progressively damaged the acquisition device. It could not be replaced before the end of 2007.

Last, we may notice a jump amounting $8 \mathrm{nT}$ in $\mathrm{dF}$ due to an accidental move of the scalar magnetometer which was put again on its previous place in December.

In order to evaluate the accuracy improvement due to the base line correction, we compared the observed secular variation with the secular variation predicted by the IGRF10 model. Figure 11 shows that the agreement between observed and predicted values is fairly good for the vertical component and total field, but not for the horizontal components. Howev- 


\section{DMC}
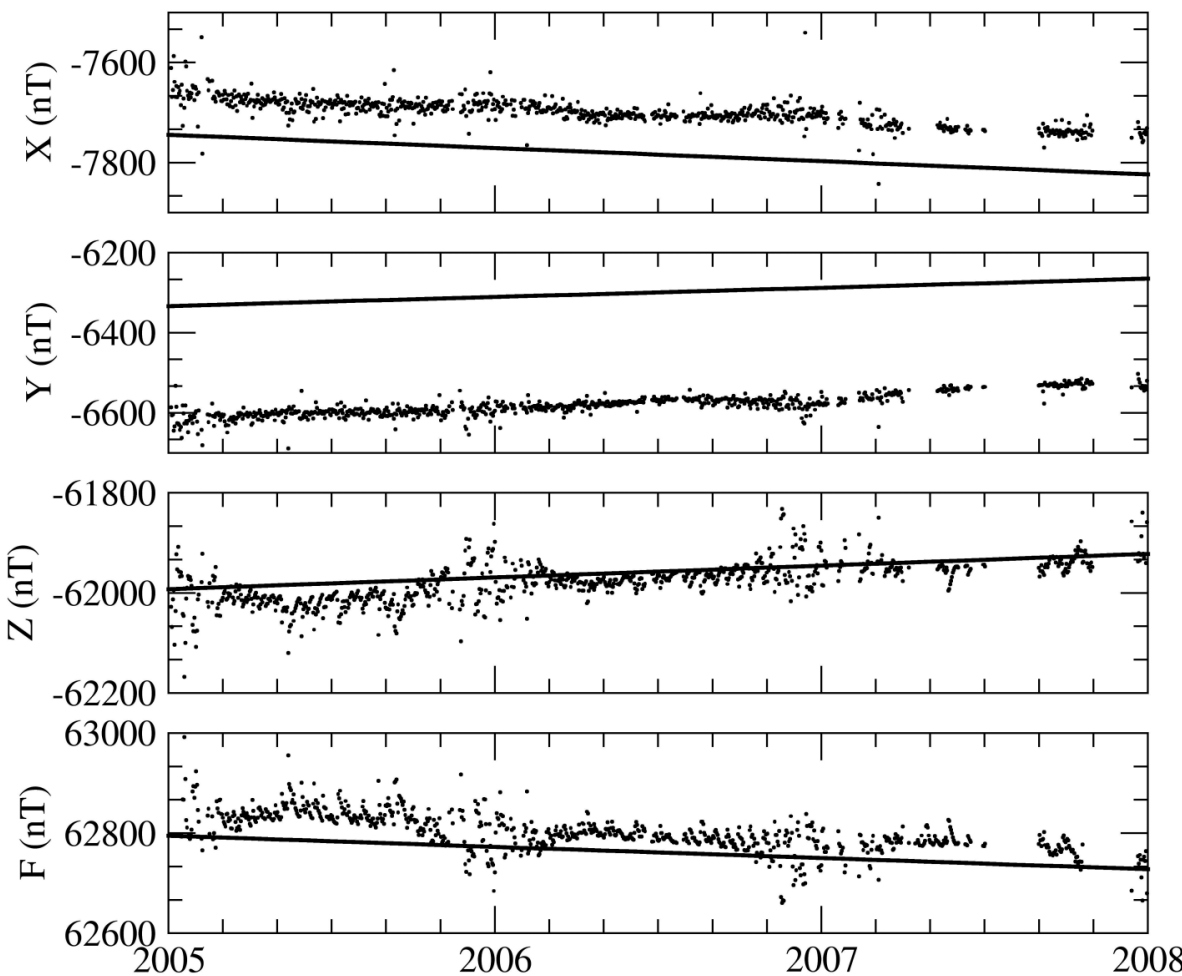

Fig. 11. Comparison of the daily mean values of the absolute field with the field predicted by IGRF10 model. Note the fairly good agreement for components $\mathrm{Z}$ and $\mathrm{F}$ and the discrepancy for $\mathrm{X}$ and $\mathrm{Y}$ components, amounting around 80 and $-270 \mathrm{nT}$ respectively (observed minus predicted values). In any case, there is a good agreement in secular variation.

er, the discrepancy is constant, and hence, the predicted and calculated secular variations are in good agreement. Bearing in mind the behaviour of the base lines and, in addition, the uncertainty on the azimuth mark orientation with respect to the true North, we might suspect a declination error. But an elementary calculation shows that a constant angular error cannot explain the constant shifts, all the more as $\mathrm{X}$ and $\mathrm{Y}$ do not vary in the same way. Although further controls are desirable, especially with regional models like ARM (i.e. Gaya-Piqué et al., 2006) we are confident in the relevance of the corrected, absolute field values yielded by the combination of adopted base lines and magnetometer outputs.

\section{Future improvements and conclusions}

Despite the numerous, partly unforeseen difficulties, the progress towards a high standard observatory is encouraging. Three main difficulties remain to be overcome: a) proper temperature measurements and control; b) power supply stabilization; c) explanation and remedy to the base line drift. A solution should be brought to the two first points thanks to the deployment of a new acquisition which will incorporate temperature recording elements and will be powered by batteries which should stand main supply cuts and damp its fluctuations. The third point is more difficult to tackle. As the absolute measurements could luckily be resumed, 
we will be able to follow the base line drift more closely. The analysis of the piecewise previous data should provide an additional clue. On the other hand, technical suggestions are the set up of tiltmeters and the installation of another magnetometer of a different type, which is possible without difficulty on a second, at the moment unused pillar.

The data collected within these three first years of permanent opening show that it makes sense to deploy an observatory on the ice cap despite the potential disturbances which might be caused by the ice drift and despite the unusual environmental conditions. It is too early to evaluate the impact of these data on the knowledge of the internal and external Earth's field, although some preliminary studies have already shown their importance (Lepidi et al., 2003; Cafarella et al., 2007). This paper focuses on the long-term field variation, whose validation is of prime importance for the internal field knowledge, but we have to mention the collection of the one second data, not yet worked out. Along with the high frequency data recorded with induction coils in a project conducted in parallel by 1'Aquila University, these data should be very helpful in the realm of solar-terrestrial physics. Last, due to the exceptional environmental conditions, the observatory deployed at DomeC offers very attractive facilities for testing magnetometers intended for planetary exploration.

But in order to strengthen the position of the magnetic observatory project, to improve the routinely working and data quality, and finally to make the observatory recognized as a high standard one, unfortunate decisions like the one mentioned in Section 3.1 should be avoided in the future and a dedicated and educated observer should be devoted to the observatory operation.

\section{Acknowledgements}

The authors express their thanks to J.M. Torta and S. Marsal who critically reviewed the manuscript and made helpful suggestions for improving it. Three of us (P. Bordais, L. Agnoletto, P. Di Felice) operated the observatory during their one year term at Concordia. The financial and logistic support of IPEV and ENEA are vital for the carrying out of the project. We would like to thank them for this support. This is a contribution of C.N.R.S. UMR 7516.

\section{REFERENCES}

Cafarella, L., D. Di Mauro, S. Lepidi, A. Meloni, M. Pietrolungo, L. Santarelli and J.J. Schott (2007): Daily variation at Concordia station (Antarctica) and its dependence on IMF conditions, Annales Geophysicae, 25, 2045-2051.

De SAnTis, A., J.M. Torta and L.R. Gaya-Pique (2002): The first Antarctic geomagnetic reference model (ARM), Geophys. Res. Lett., 29 (8), doi: 10.1029/2002GL0144675.

Gaya-Pique, L.R., D. Ravat, A. De Santis and J.M. Torta (2006): New model alternatives for improving the representation of the core magnetic field of Antarctica, Antarctic Science, 18 (1), doi: 10.1017/SO954102006000095.

JANKOWSKI, J. and C. SUCKSDORFF (1996): Guide for magnetic measurements and observatory practice, (International Association of Geomagnetism and Aeronomy), pp. 235.

Lepidi, S., L. Cafarella, P. Francia, A. Meloni, P. PalanGIO and J.J. SchOTT (2003): Low frequency geomagnetic field variations at Dome C (Antarctica), Annales Geophysicae, 21, 923-932.

MandeA, M. and B. LANGlais (2002): Observatory crustal magnetic biases during MAGSAT and Orsted satellite missions, Geophys. Res. Lett., 29 (15), doi: 10.1029/2001GL013693.

NewitT, L.R., C.E. BARTon and J. BitTerly (1996): Guide for magnetic repeat station surveys, (International Association of Geomagnetism and Aeronomy, Working Group V-8), pp. 111.

Sapunov, V., A. Denisov, O. Denisova and D. Saveliev (2001): Proton and Overhauser magnetometers technology, Contributions to Geophysics \& Geodesy, Vol. 31, pp. 119-124.

SchotT, J.J. and J.L. RAsson (2007): Observatories in Antarctica, in Encyclopedia of Geomagnetism and Paleomagnetism, edited by D. GubBIns and E. HerReroBERVERA, pp. 1054.

Schott, J.J., D. Di Mauro, A. Peres, L. Cafarella, L. Magno, A. Zirizotti and A. Meloni (2005): Towards the opening of a magnetic observatory at DomeC (Antarctica), Proceedings XIth Workshop on Geomagnetic Observatories, Instruments, Data Acquisition and Processing, (Kakioka, November 17-24, 2004).

Seber, G.A.F. (1977): Linear Regression Analysis, (John Wiley and Sons (Eds), pp. 465.

Silverman, B.W. (1985): Some aspects of the spline smoothing approach to non-parametric regression curve fitting, J.R. Statist. Soc. B, 47 (1), 1-52.

TorTA, J.M., A. De SANTIS, M. ChiAPPINI and R.R.B. von FRESE (2002): A model of the secular change of the geomagnetic field for Antarctica, Tectonophysics, 347, 179-187. 